\title{
Acausality Alleviation via Nonlinear Future Prediction in Feedforward Control of Vibrations
}

\author{
Venkata R. Sonti and Anindya Chatterjee \\ Department of Mechanical Engineering, Indian Institute of Science, Bangalore 560 012, India
}

(Received 5 May 2003; accepted 14 July 2003)

\begin{abstract}
An augmented version of the LMS feedforward algorithm is used, in discrete time simulation, to control a twobeam and one-spring system in an acausal configuration. For perfect control, the required control force is unbounded due to acausality. Imperfect but good control is possible if about-to-act disturbances are known slightly in advance. In the simulations, a broadband yet deterministic chaotic time series is used as the disturbance (a brief characterisation of measured fan noise is presented for comparison). Signal determinism is exploited using a neural network trained to predict some future values of the signal, and the prediction is included in the feedforward loop of an augmented version of the filtered-X LMS algorithm. This controller significantly outperforms the standard algorithm without future prediction. The present controller can be viewed as a neural network based and disturbance-dependent approximation to an acausal controller; but also as a causal nonlinear controller for a linear system in an acausal configuration.
\end{abstract}

\section{INTRODUCTION}

We present a new nonlinear active feedforward vibration controller that exploits any determinism present in broadband disturbances acting on the system. The controller uses a neural network to predict future values of the disturbance, and uses these predicted future values to offset the problems encountered due to acausality. Except for these predictions, which are made using a nonlinear technique, the controller is completely structured around the standard LMS algorithm, and inherits its well-known strengths. The controller is developed for a system of two coupled beams; using this system for demonstration, some issues related to possible control strategies in the presence of acausality are addressed.

Active noise and vibration control using the feedforward LMS algorithm and its variations has been a topic of research for almost four decades. Of this, much research has focused on sinusoidal disturbances at single or multiple frequencies, and some on random signals. In this paper, we consider signals that, though broadband, have a certain degree of predictability or determinism in the short term.

Figure 1 shows a system disturbed by a force $F(t)$ at point $\mathrm{C}$ and controlled by force $G(t)$ at point $\mathrm{A}$. The aim is to minimise the response $x(t)$ at point $\mathrm{B}$. In linear feedforward control, $G(t)=\mathcal{L}(F(t))$, where the operator $\mathcal{L}$ represents an adaptively refined linear filter. Where feedforward control fails to achieve perfect control of $x(t) \equiv 0$, a possible reason is acausality, as described below.

Acausality is best illustrated by an acoustics example. Let Fig. 1 represent the inside of an anechoic chamber. Let $F(t)$ and $G(t)$ be the outputs of audio speakers. Now, if distance $\mathrm{AB}>\mathrm{BC}$, then the controller must anticipate the future of the disturbance. Such a controller is termed acausal. The same issues arise, though less transparently, in vibrations.

If $F(t)$ is a sinusoid, then its future is known and acausality is not a problem. If $F(t)$ is random (broadband and stochastic), then its future cannot be predicted and perfect control is not possible. The LMS algorithm provides some vibration cancellation, but we aim to outperform it. Other than plain LMS, the main approach so far to feedforward control under broadband disturbance is based on stochastic modelling of the disturbance and minimising the expected value of the squared response. ${ }^{1}$

In this paper, we propose to tackle the broadband disturbance by directly exploiting any significantly deterministic behaviour present in it. Our approach therefore leaves out high dimensional broadband disturbances such as fan noise, which we discuss below. For the present work, however, we primarily use a time series from a well known low-dimensional chaotic system, the future values of which can be reasonably predicted using a neural network based modelling approach. These predicted values are then used in an acausal LMS algorithm, which requires some future values of the disturbance.

Thus, our controller is really a causal but nonlinear one. However, our understanding of it is based on viewing it in two parts. The first part is a neural network that predicts some future values of the disturbance, and the second part uses these future values in a linear but acausal controller.

\section{PRIOR WORK}

In the literature, there is a large amount of work reported on control of tonal disturbances (sinusoids and linear combinations thereof), but relatively less on the control of broadband disturbances. One of the early efforts in broadband noise control is by Ross. ${ }^{2}$ A fixed (nonadaptive) causal controller was constructed using theoretical transfer functions and implemented for downstream cancellation of noise in a 1-D duct application below the first cut-on frequency. Reductions of 15-20 dB were obtained below $350 \mathrm{~Hz}$. Next, Ross ${ }^{3}$ implemented the same system using an adaptive off-line procedure. Several practical difficulties in the experiment were overcome and about $11 \mathrm{~dB}$ of control over a range of $120 \mathrm{~Hz}$ was shown. Fontaine and Shepherd ${ }^{4}$ implemented a controller, again in a 1-D duct application, by manually adjusting delays and filters. Feedback issues were taken care of and 15$20 \mathrm{~dB}$ of reduction up to $650 \mathrm{~Hz}$ was obtained. Roure ${ }^{5} \mathrm{dem}-$ onstrated broadband noise control in a duct using an adaptive 